\title{
EXPERIENCE WITH INDUCTIVE COUPLED CARDIAC PACEMAKERS
}

\author{
L. D. Abrams \\ Department of Surgery, The University of Birmingham and The United Birmingham Hospitals, \\ Queen Elizabeth Hospital, Birmingham, England
}

\section{J. C. Norman}

The Department of Surgery, University Hospital and The University of Michigan Medical Center, Ann Arbor, Mich.

In June, 1960, Abrams, Hudson, and Lightwood ${ }^{1}$ reported the use of the inductive coupled cardiac pacemaker for the treatment of three patients with complete heart block. The method is illustrated in Figure 1. A pulse generator is attached by a flexible lead to an external primary coil that is strapped to the skin over the implanted secondary coil, the ends of which are the myocardial electrodes. The pulse in the primary coil produces a pulse in the secondary coil by simple electromagnetic induction. The secondary coil consists of a thousand turns of 38 gauge standard copper wire wound to form a ring with an inner apperture of $2 \mathrm{~cm}$. The ends of the wire are welded to braided No. 26 gauge stainless steel leads armed with atraumatic needles. The coil and leads are covered by silicone rubber.

The appearance of these coils in a patient is shown in FIGURE 2, which is a lateral chest X-ray taken six days after the operation. The lead from the pulse generator is seen going to a solid external coil that overlies the subcutaneous ringshaped secondary coil. The secondary coil must be firmly fixed in a position in which it has a stable base. In patients who are not fat the best place is on the front of the left third intercostal space, but if there is a considerable depth of subcutaneous fat the internal coil is placed on the front of the sternum. The leads are laid in a full turn around the coil before being passed back through the intercostal space to the heart, which is exposed through an anterior fourth space thoracotomy. The silicone rubber can be peeled off the stainless steel wire so that the right length to form a sweeping curve between the chest wall and the area on the heart chosen for the implantation of the electrodes can be used. The electrodes are fixed by taking three one centimeter bites with the atraumatic needle so that they all fall in an area of myocardium about $1 \mathrm{~cm}$. by $0.6 \mathrm{~cm}$. The insulation is pulled right up to the muscle, and none of the wire should show through the surface. The excess wire is cut off under slight tension so that the end retracts into the myocardium; thus the lead itself forms the electrode. A patch of Teflon felt, $2.5 \mathrm{~cm}$. long by $1 \mathrm{~cm}$. wide, is sutured to the heart, covering the area containing the electrode and holding the first centimeter of the insulated lead.

Figure 3 shows the internal wires in the postero-anterior X-ray. The loop at the end is the buried wire.

FIGURE 4 shows the patient on the sixth postoperative day wearing his coil and pacemaker. The strapping on the lower part of the chest covers temporary thin, braided wire, direct pacemaking electrodes, which are always put in so that direct pacemaking can be used while any postoperative swelling and tenderness settles down and the patient becomes accustomed to the use of the inductive coupled system. This patient, who was 41 and had had many Stokes-Adams attacks every 
Abrams \& Norman: Inductive Coupled Pacemakers 1031

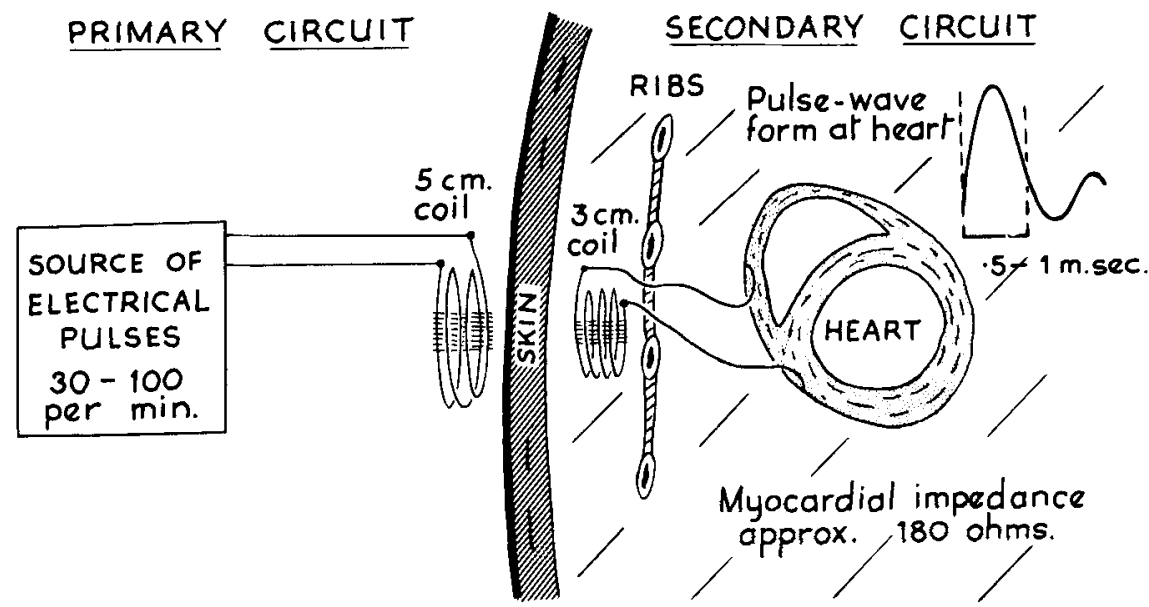

Figure 1. The principle of the inductive coupled pacemaker (by permission of The Lancet).

FIGURE 2. Lateral X-ray of patient showing primary and secondary coils. 
day for some time, was confident in his use of the apparatus at this stage; the direct wires were removed a few days later.

The system has the following advantages: The implant is of the simplest possible construction consisting essentially of a length of wire. The problems of the electrodes and the encapsulation are common to all buried pacemakers; they are the only buried problems in this system. The internal coil is robust and can be autoclaved. There are no electronic components to fail. The pacemaker itself is external so there is no difficulty in changing it for any reason. Rate and power controls can be incorporated. Moreover pacemaking is voluntary and can be stopped if the patient reverts to stable sinus rhythm, as has happened in three of our cases.

These advantages must be balanced against the disadvantages of having to carry an external pacemaker and to wear the external coil strapped to the skin. Experience with 40 patients has shown that these disadvantages are not great. There has been very little trouble in maintaining the inductive coupling even during violent movements of the arms, and the patients appreciate the ease with which their pacemaker can be changed. The electronic engineers we have operated on have been especially appreciative of this facility and have expressed their relief when told that the pacemaker itself was not to be buried.

Of the 40 patients, eight have died very shortly after the operation with their pacemakers working. Two of these developed myocardial infarction; the others,

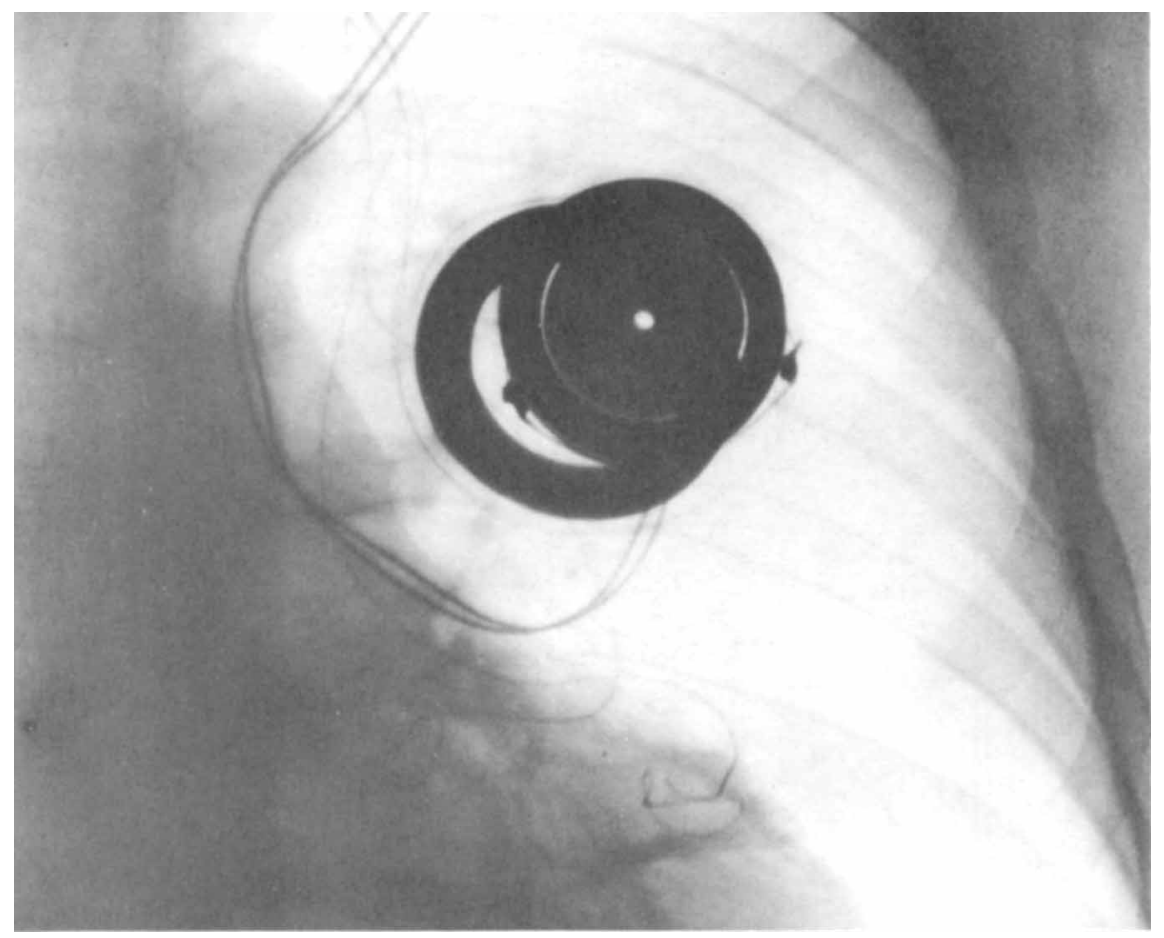

Figure 3. Postero-anterior X-ray showing coils and leads. The loops in the heart shadow form the electrodes. 
all in old age, died of combination of respiratory and cardiac failure. One patient died of ventricular tachycardia, which did not respond to treatment, and two have had sudden ventricular fibrillation demonstrated by electrocardiogragh-after the prompt institution of external cardiac massage; neither was resuscitated.

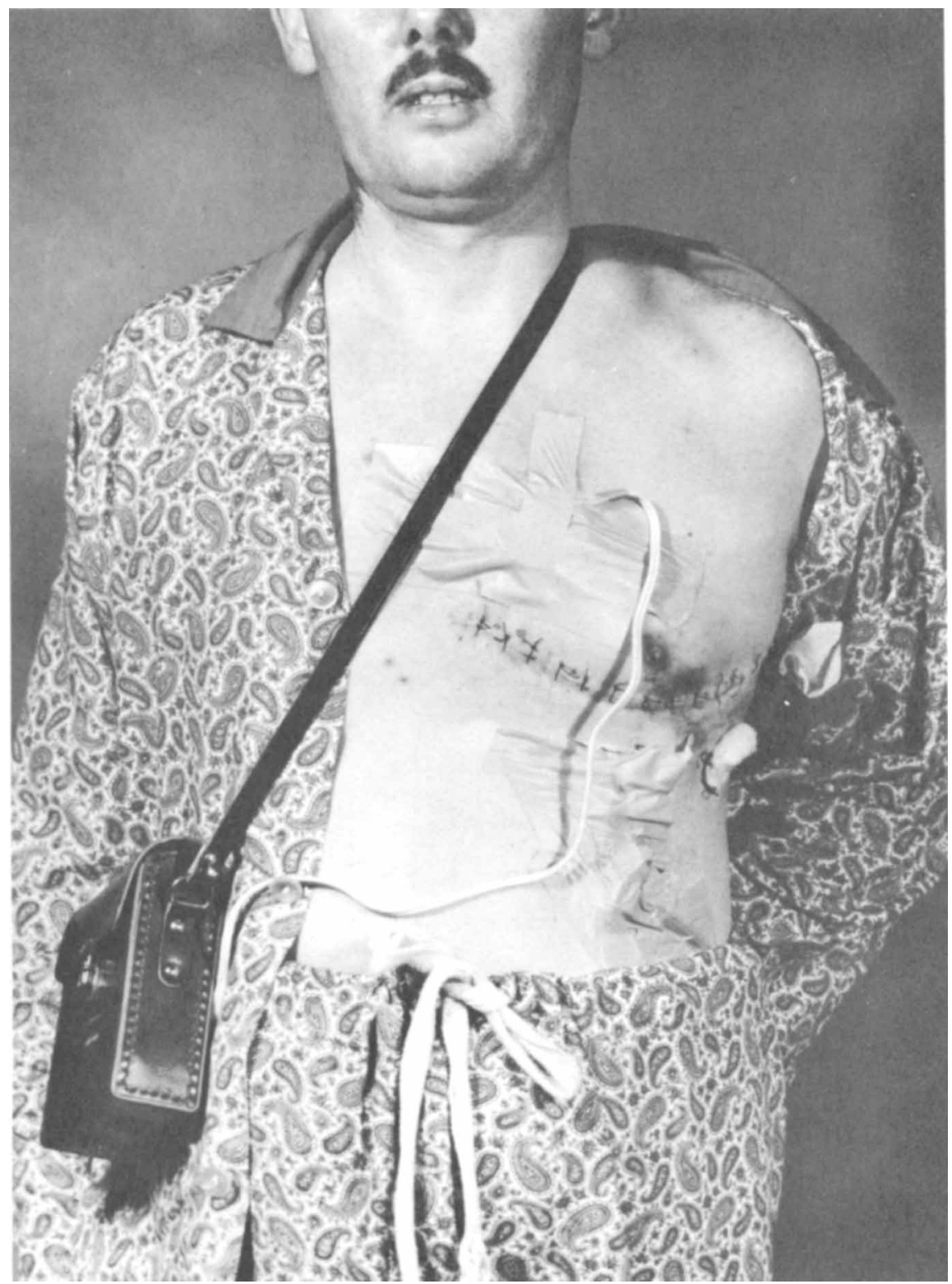

Figure 4. A 41-year-old electronic engineer on the 6th postoperative day. 

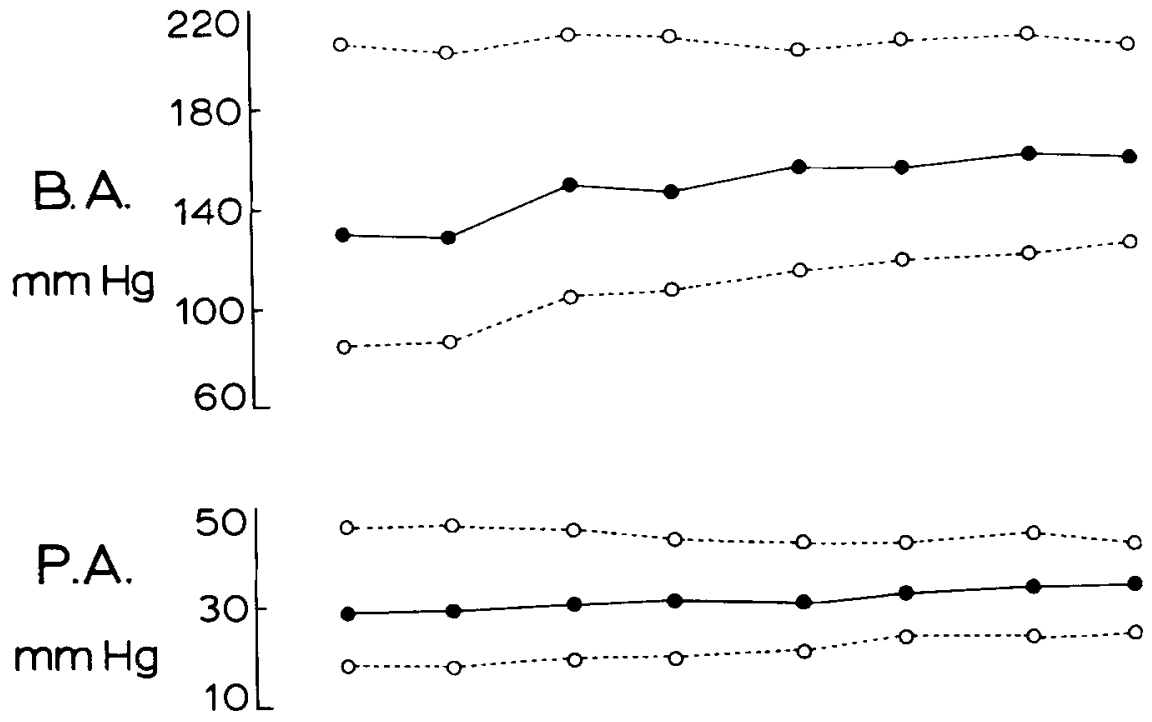

$\begin{array}{cc}\text { P.W. } & 30 \\ \mathrm{~mm} \mathrm{Hg} & 10\end{array}$

$\begin{array}{ll}\text { INDEX }^{3} & \\ \mathrm{l} / \mathrm{min} / \mathrm{m}^{2} & 1\end{array}$
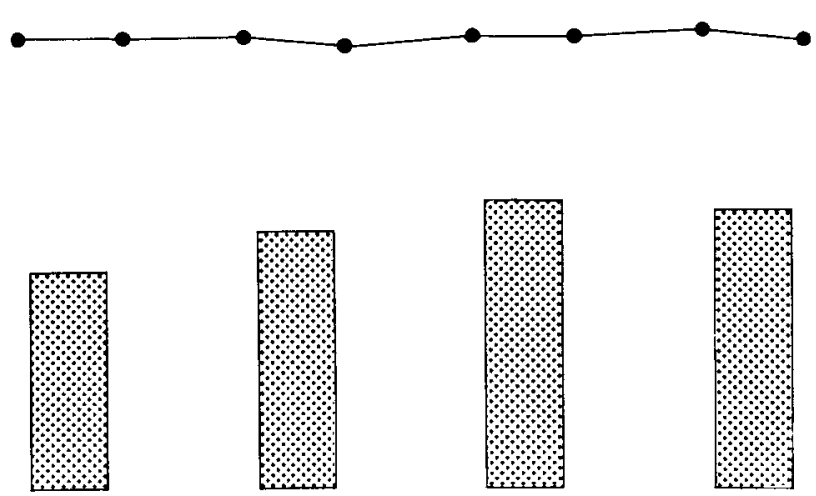

I.V.R.

$50-65$

$70-83$

$85-100$

\section{HEART RATE}

Figure 5. Mean values obtained from hemodynamic study of 14 patients at rest.

Four patients have died in hospitals from other conditions, having been paced for four months in two cases, twelve months in the third, and thirty-two months in the fourth. Seven other patients have also died, having been paced for periods varying from four to sixteen months. Three of these deaths were due to pacemaking failure, but the other four patients died during their sleep at home and the cause was not determined. 


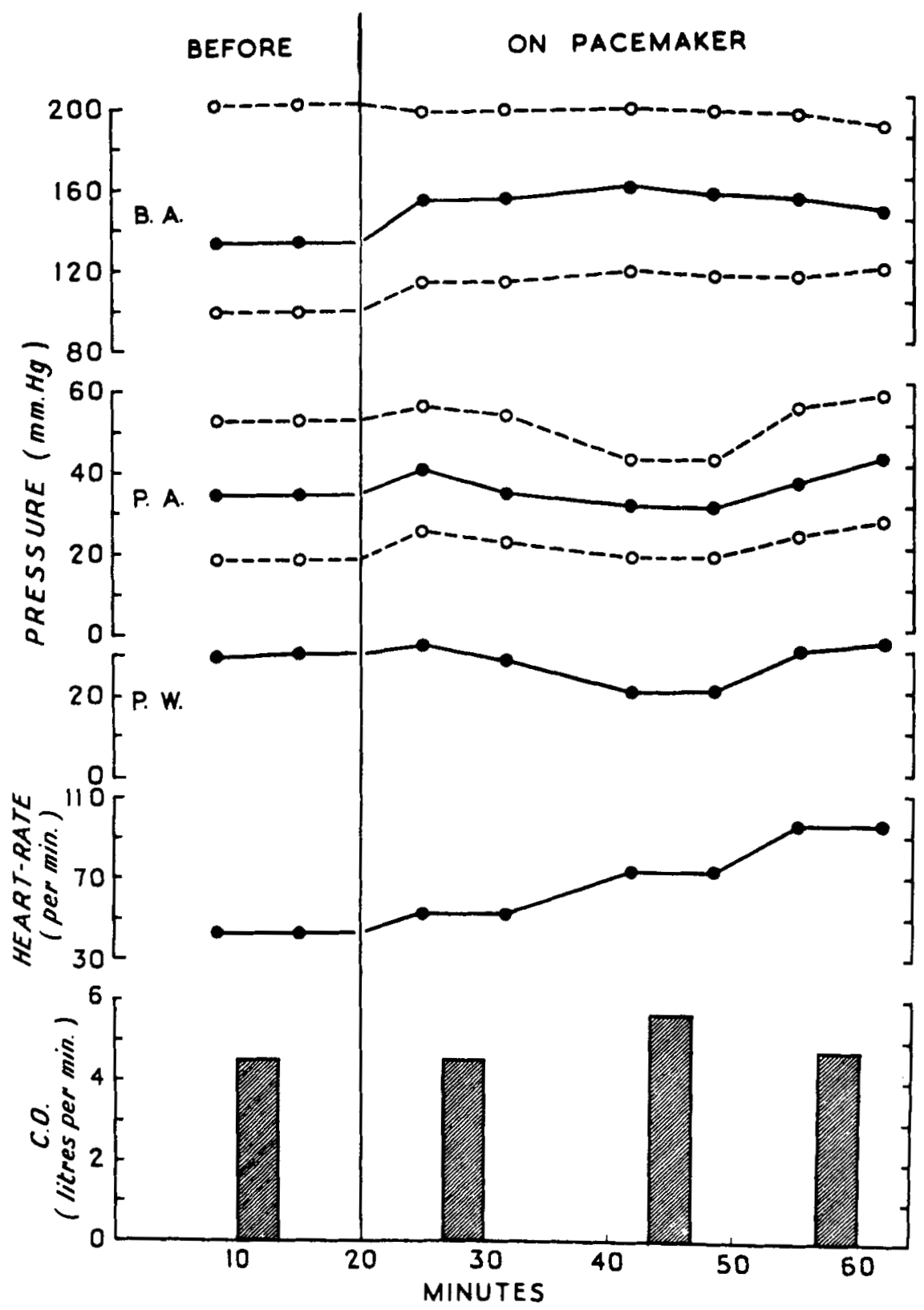

FIGURE 6. Hemodynamic study of patient in heart failure showing increase in output and reduction of pulmonary wedge pressure at 70 beats per minute. Two estimations at each heart rate (by permission of The Lancet). 
The remaining nineteen patients are still alive and well, having been paced for periods varying from three months to three years and five months. They lead normal lives. The men work and dig their gardens, the ladies look after their houses and go out dancing; the pacemaker is not waterproof so they cannot swim, but it can be placed outside the bath.

Segel, Hudson, Harris, and Bishop ${ }^{2}$ of the Department of Medicine, University of Birmingham, England, have made hemodynamic studies of 14 of our patients with complete heart block. They have measured the cardiac output by the direct Frick method at idioventricular rate and various pacemade rates, both at rest and during exercise, in those patients who could exercise. It can be seen from FIGURE 5 , which shows the mean values of the 14 cases studied at rest, that the maximum cardiac output is obtained between 70 and 83 beats per minute. It appears, therefore, that even for the adequate treatment of patients at rest it is necessary to have a variable rate instrument, if the object of treatment is to be more than the prevention of Stokes-Adams attacks. A large proportion of our cases have had low cardiac output, and some of these studies were undertaken to evaluate pacemaking in patients who were severely disabled by low cardiac output but did not have Stokes-Adams attacks.

One such case was reported in 1960, and the results of the hemodynamic study are shown in FIGURE 6. Pacemaking was carried out with an intracardiac catheter electrode. At idioventricular rate his cardiac output was 4.5 liters per minute and the pulmonary wedge pressure nearly $30 \mathrm{~mm}$. of mercury; an increase of rate to 60 beats per minute did not improve his cardiac output; but when the rate was increased to 70 there was an improvement in cardiac output to 5.6 liters per minute; and it should be noted that his wedge pressure fell to $20 \mathrm{~mm}$. of mercury; this was accompanied by a dramatic relief of breathlessness. Over the course of the next few days the patient was paced out of cardiac failure. He was the third patient to have an inductive coupled pacemaker inserted, and he has been paced by this method ever since the end of April, 1960. He is a retired engineer who has helped us greatly over the past three years by testing new apparatus and giving us very useful advice on the convenience of various designs of external coil and pacemaker.

Several of our patients have found that it is helpful to increase their heart rate with exercise-this not only reduces breathlessness but makes them feel more comfortable in a way that they do not seem to be able to define accurately. Some of the patients like to decrease their heart rate when they settle down in bed to go to sleep; again the sensation is ill-defined. There is, of course, no sensation whatsoever from the pacemaker itself, but patients feel more comfortable under certain conditions with different heart rates. A lot of these patients are conscious of the beat of the heart, especially those whose hearts have been stopping now and again for some time.

Nearly all our patients have used a pacemaker, shown on the left of FIGURE 7, containing a rechargeable battery. The patient has two pacemakers and a charger so that one pacemaker can be on charge while the other is used. It is also possible to charge the pacemaker while it is in use, and it can be completely charged at night, during which time a sufficient charge is gained to carry on throughout the day. The lead to the external coil is on a plug so it can be changed from one pacemaker to the other easily and quickly.

If it is necessary to change the external coil, the strapping is removed while the coil is held in position. The spare external unit attached to the spare pacemaker is quickly exchanged for the first coil and strapped into position at leisure. 


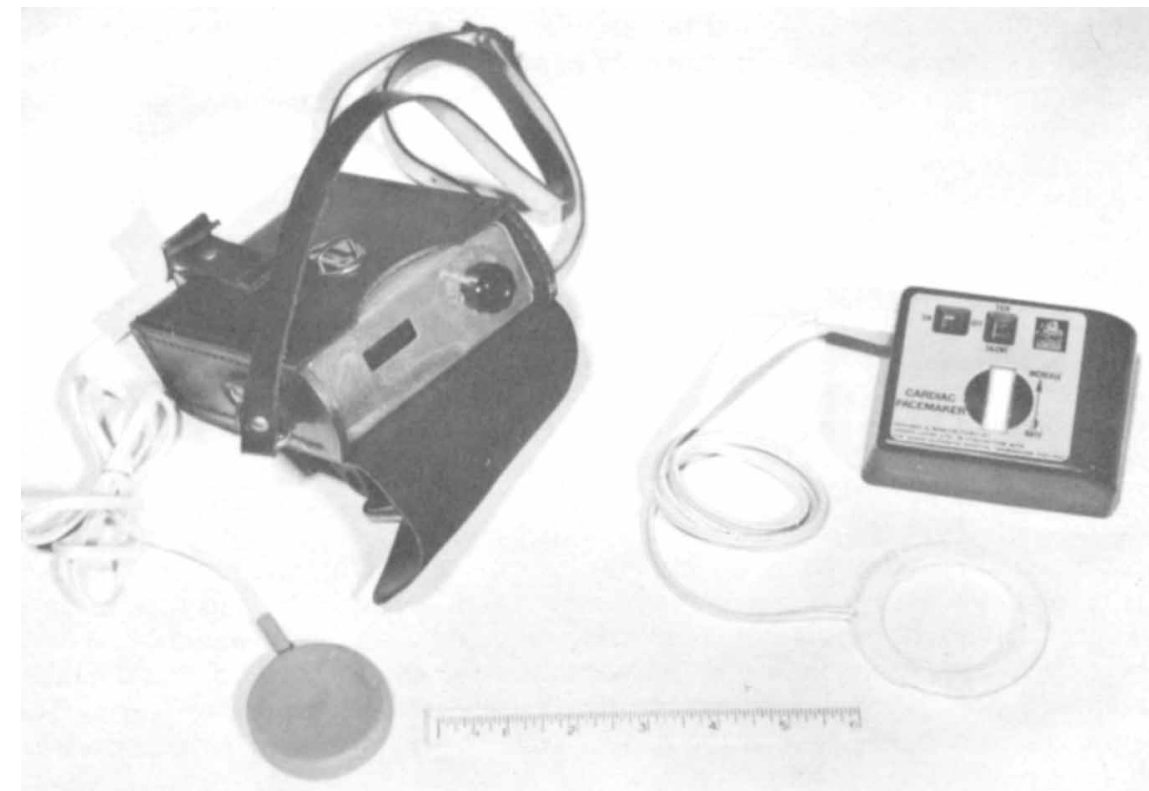

FIGURE 7. Left: Pacemaker and external coil used from late 1960 until mid 1963. Right: New pacemaker and external coil.

The patients are all instructed to reduce their pacemade rate before changing apparatus, although this can be done so quickly that there is little chance of anything going wrong. However, if pacemaking is stopped suddenly at a high rate there is a slight risk of the patient having a Stokes-Adams attack if pacemaking is not resumed immdiately. This tendency seems to be greater immediately after pacemaking is started and does not decrease until one or two weeks thereafter.

If the pacemaker is set at a rate below the idioventricular rate before changes are made, the tendency to have Stokes-Adams attacks vanishes. It has been our experience that patients who have been paced for periods longer than two or three months always show a very greatly reduced tendency to have Stokes-Adams attacks. We have studied this by deliberately turning off the pacemaker for varying periods under careful supervision.

We are now bringing into use the pacemaker shown on the right of FIGURE 7. This is a very much more compact and powerful instrument than the old model. The interior is shown in FIGURE 8 , and it will be seen that the battery is a standard $\mathrm{U} 2$ torch battery; this is one of the most popular batteries in the world and is available at all points eastward from Alaska to Siberja. It can be changed in less than five seconds, and one of the cheaper varieties will pace our patient with the highest power required for more than a week. There is a rate control that is easy to adjust but difficult to move accidentally. There is an on-off switch and also a switch that enables a tick to be heard with each pulse. To start with patients are very comforted by hearing their pacemaker in action. The tick is not produced if a pacemaking pulse is not supplied to the coil. With familiarity they prefer to turn the tick off. 
The pacemaker makes a loud noise if the external coil is not properly connected; this also serves as a warning should trouble develop with the external coil lead. When this does occur it is usually heralded by intermittent pacemaking due to fracture of part of the wire in the flexible lead. This gives rise to an intermittent noise even if the patient does not notice the change in pacemaking, which he well may not. However he has an indication to change the external coil. The power control, high and low, is the switch seen in the upper part of the mechanism. The low range will, in fact, provide sufficient power to pace the great majority of patients for more than eighteen months.

We find that the power requirements increase fairly rapidly, although not to a great degree, during the first two weeks. Thereafter there seems to be very little increase for several months. We have a very good indication of the power requirement because it can be estimated from the degree of separation of primary and secondary coils that can be tolerated before pacemaking stops. The first thing the patient notices is an irregularity of heart beat during vigorous movement because of slight movement of the external coil; this occurs some time before pacemaking becomes really difficult, and a more powerful pacemaker is needed. It is for this reason that the power change switch is inside the pacemaker, since we feel that no more power than necessary should be used. All patients who have been paced for more than eighteen months have shown need for considerably increased power, roughly equivalent to six volts from a direct pacemaker. The circuit is so designed that as the battery runs down the rate of pulse decreases, but the pulse power remains constant.

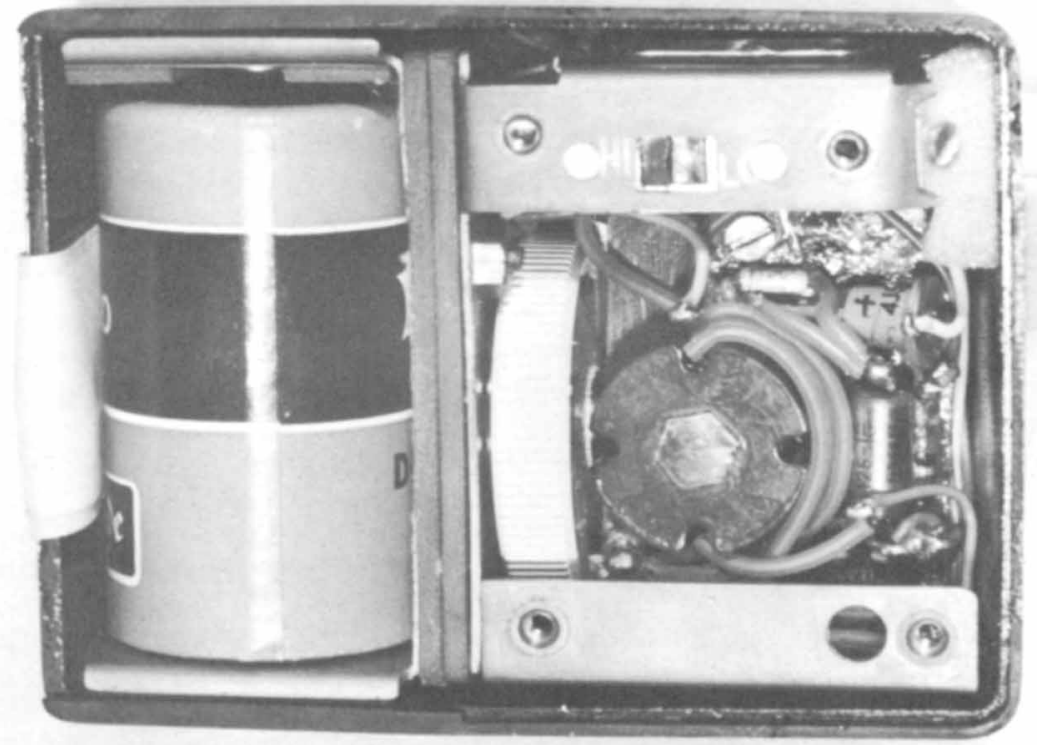

FIGURE 8. Interior of the new pacemaker. The U 2 battery on the left. "HI-LO" switch at top of mechanism. 


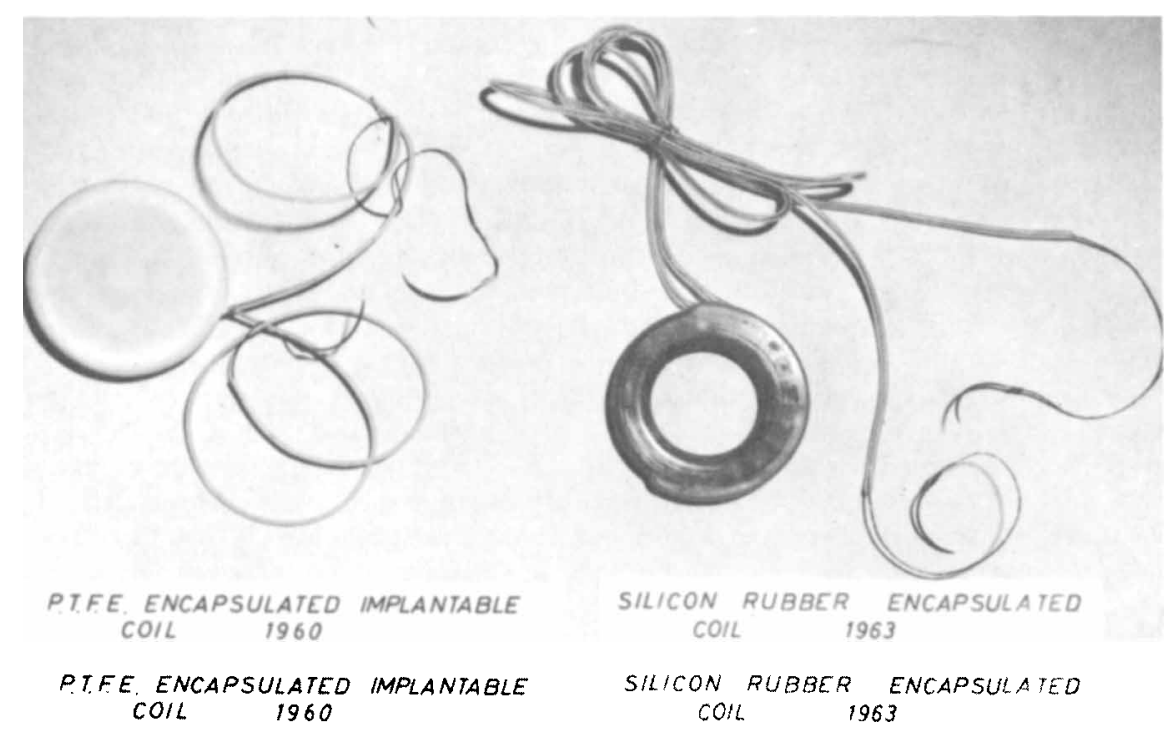

Figure 9. Old and new types of secondary coil.

We have used various designs of external coil and now think that a simple ring, slightly wider than the internal coil, without any core gives the best coupling.

Apart from the very considerable improvements in the design of the pulse generator itself, the most significant improvements have been in the design of the internal coil. The first coils were home made, wound on nylon bobbins, and with rather thin, braided stainless steel electrodes. These fractured and the coils were replaced. All but the first three patients have had 26 gauge braided stainless steel electrodes, and these have proved very satisfactory. Inductive coupling has this advantage, that the pulse must be both positive and negative so that electrolytic effects should be minimized. We have had the opportunity of examining this wire when it has been used for pacemaking for sixteen months, and we are assured by the Department of Metallurgy of the University of Birmingham, England, that the appearance of the wire is practically unchanged when compared with new wire under a microscope. We have had coil failure due to breakdown of the encapsulation; this has only occurred with the coil we used from late 1960 to early 1962. This type and the new coil are shown in FIGURE 9. These coils were encapsulated in Teflon and were formed at high temperature and pressure, which set up stresses in the coil. These resulted in hair line cracks that allowed the entry of body fluids and corrosion of the copper wire. The solid coil was rather difficult to fix accurately on the anterior chest wall, which is an essential part of the technique. It was necessary to make an incision high in the pectoral muscle so that a bed was formed for the coil, and then a piece of thin Teflon cloth was sutured over it to the margins of the defect in the muscle, thus giving accurate location. The new coil is an open ring and is placed in a subcutaneous pocket, closed with a few 0000 silk sutures, above the wound. Tissues grow through the 
center of the coil, which shows no tendency to move at all. There is considerably less postoperative swelling around the new coils, and they are more efficient magnetically. We have had fractures of the 26 gauge wire in four patients, but they have all been due to mistakes in placing the leads. In one of the first coils, using this wire, the leads were too short, and they were taken straight back through the intercostal space from the coil; this caused fracture at the junction of the lead and the coil. In two patients one of the leads fractured very close to the heart; at the second operation it was found that the lead and its insulation were not supported by the Teflon felt patch used to cover the embedded wire and a short length of the adjacent lead; if this is not done then movement is concentrated on the small portion of bare wire between the insulation and the heart, causing fatigue fracture. We now think it is likely that the rather stiff Teflon insulation of these particular wires also gave rise to a tendency for concentration of movement on any soft spot and therefore increased the likelihood of fatigue fracture; there has been no trouble with silicone rubber insulated coils and leads, which have been used for over a year.

In conclusion, we may say that this system has now been in use for three and a half years. Although the patients have to be instructed and must learn the technique of using the apparatus, they seem to have very little trouble with their heart aids and have been enabled to lead useful lives for periods exceeding two years in several cases and three years and five months in one case. We think this is sufficient justification for the continued use of the inductive coupled pacemaker.

\section{Acknowledgments}

We wish to thank Mr. R. Lightwood (Electronics Engineer, Queen Elizabeth Hospital, Birmingham, England) and Joseph Lucas, Ltd., Birmingham, England, for their work in developing the pacemaking apparatus.

We are very grateful to the Department of Metallurgy, University of Birmingham, England, for examining the stainless steel wire.

We thank Mr. T. F. Dee for the photographs and the Editor of The Lancet for permission to reproduce FIGURES 1 and 6 .

\section{References}

1. Abrams, L. D., W. A. Hudson \& R. Lightwood. 1960. The Lancet i: 1372.

2. Segal, N., W. A. Hudson, P. Harris \& J. M. Bishop. The Circulatory Effects of Electrically Induced Changes in Ventricular Rate at Rest and During Exercise in Complete Heart-Block. In press. 